\title{
A PROBABILITY DISTRIBUTION ASSOCIATED WITH THE HURWITZ ZETA FUNCTION
}

\author{
R. L. DUNCAN
}

\begin{abstract}
A new discrete probability distribution is defined in terms of the Hurwitz zeta function and its relationship to the Poisson distribution is demonstrated.
\end{abstract}

If we define the Hurwitz zeta function by

$$
\varsigma(s, a)=\sum_{n=0}^{\infty} \frac{1}{(n+a)^{s}}
$$

for $a>0$ and $s>1$, then it is easy to show that

$$
f(x ; a)=a_{\zeta}(x+1, a+1), \quad x=1,2,3, \ldots,
$$

is a probability density function (p.d.f) with factorial moment generating function

$$
G(t)=a \sum_{n=1}^{\infty}\left(\frac{1}{n+a-t}-\frac{1}{n+a}\right)
$$

for $|t|<1+a$ and factorial moments

$$
G^{(m)}(1)=a m ! \zeta(m+1, a) .
$$

It will now be shown how $f(x ; a)$ arises in a natural way from the Poisson distribution. It is known $[\mathbf{1}, \mathbf{1 3 . 1 2}]$ that

$$
\varsigma(s, a)=\frac{1}{\Gamma(s)} \int_{0}^{\infty} \frac{y^{s-1} e^{-a y}}{1-e^{-y}} d y .
$$

If $g(x ; y)$ and $h(y ; a)$ are the p.d.f.s for the Poisson distribution with mean $y$ and the exponential distribution with mean $1 / a$, then

$$
f(x ; a)=\int_{0}^{\infty} g^{*}(x ; y) h(y ; a) d y
$$

where

$$
g^{*}(x ; y)=\frac{g(x ; y)}{1-g(0 ; y)}, \quad x=1,2,3, \ldots
$$

is the p.d.f. for a truncated Poisson distribution. Thus, by the same argument used in [2, 5.13B], $f(x ; a)$ is the p.d.f. for a compound truncated Poisson distribution where the parameter in the truncated Poisson distribution has an exponential distribution with mean $1 / a$.

Received by the editors November 22, 1985 and, in revised form, February 17, 1986 and May 21, 1986.

1980 Mathematics Subject Classification (1985 Revision). Primary 62E10. 
Now let $a$ and $b$ be positive and replace $h(y ; a)$ by the p.d.f. for the gamma distribution defined by

$$
h(y ; a, b)=\frac{a^{b}}{\Gamma(b)} y^{b-1} e^{-a y}, \quad 0<y<\infty .
$$

Then it follows as before that the compound truncated Poisson distribution, where the truncated Poisson distribution is the same as above but whose parameter now has a gamma distribution, will have the p.d.f.

$$
f(x ; a, b)=a^{b} b(b+1) \cdots(b+x-1) \zeta(x+b, a+1) / x !, \quad x=1,2,3, \ldots,
$$

and it can be shown that the factorial moments for this distribution are

$$
G^{(m)}(1)=a^{b} b(b+1) \cdots(b+m-1) \zeta(m+b, a) .
$$

The corresponding problem for the compound Poisson distribution is considered in $[2,5.13 \mathrm{~B}]$.

If $b / a=c=$ constant and $x$ is a positive integer, then

$$
\varsigma(x+b, a+1)=\left(\frac{c}{b+c}\right)^{x+b} \sum_{n=0}^{\infty}\left(1+\frac{c n}{b+c}\right)^{-x-b}
$$

and

$$
f(x ; a, b)=\frac{c^{x}}{x !}\left(\frac{b}{b+c}\right)^{b}\left[\frac{b(b+1) \cdots(b+x-1)}{(b+c)^{x}}\right] \sum_{n=0}^{\infty}\left(1+\frac{c n}{b+c}\right)^{-x-b} .
$$

Hence

$$
\lim _{b \rightarrow \infty} f(x ; a, b)=\frac{c^{x} e^{-c}}{x !} \sum_{n=0}^{\infty} e^{-c n}=\frac{c^{x} e^{-c}}{x !\left(1-e^{-c}\right)} .
$$

Thus the truncated Poisson distribution with parameter $b / a$ provides a simple approximation for $f(x ; a, b)$ when $a$ and $b$ are large.

For $t<\log (1+a)$, the moment generating function is given by

$$
\begin{aligned}
M_{X}(t) & =a^{b} \sum_{n=1}^{\infty}(n+a)^{-b} \sum_{x=1}^{\infty} \frac{b(b+1) \cdots(b+x-1)}{x !}\left[\frac{e^{t}}{n+a}\right]^{x} \\
& =a^{b} \sum_{n=1}^{\infty}(n+a)^{-b}\left[\left(1-\frac{e^{t}}{n+a}\right)^{-b}-1\right] \\
& =a^{b} \sum_{n=1}^{\infty}\left[\left(n+a-e^{t}\right)^{-b}-(n+a)^{-b}\right]
\end{aligned}
$$

If $Y=(a X-b) / c$, where $c=\sqrt{b(a+1)}$, then

$$
M_{Y}(t)=\sum_{n=1}^{\infty}\left\{\left[\frac{a}{e^{t / c}\left(n+a-e^{a t / c}\right)}\right]^{b}-\left[\frac{a}{e^{t / c}(n+a)}\right]^{b}\right\}
$$

and

$$
\begin{aligned}
\lim _{b \rightarrow \infty} M_{Y}(t) & =\lim _{b \rightarrow \infty}\left[a / e^{t / c}\left(1+a-e^{a t / c}\right)\right]^{b} \\
& =\lim _{b \rightarrow \infty}\left[\left(1+t / c+t^{2} / 2 c^{2}+\cdots\right)\left(1-t / c-a t^{2} / 2 c^{2}-\cdots\right)\right]^{-b} \\
& =\lim _{b \rightarrow \infty}\left[1-t^{2} / 2 b+o(1 / b)\right]^{-b}=e^{t^{2} / 2}
\end{aligned}
$$


Hence $Y$ has an approximate standard normal distribution when $b$ is large.

If $Z=2 a X$, then

$$
M_{Z}(t)=\sum_{n=1}^{\infty}\left[\left(\frac{a}{n+a-e^{2 a t}}\right)^{b}-\left(\frac{a}{n+a}\right)^{b}\right]
$$

and

$$
\lim _{a \rightarrow 0} M_{Z}(t)=\lim _{a \rightarrow 0}\left(\frac{a}{1+a-e^{2 a t}}\right)^{b}=(1-2 t)^{-b} .
$$

Thus $Z$ has an approximate chi-square distribution with $2 b$ degrees of freedom when $a$ is small.

When $x=1$, we have

$$
\begin{aligned}
f(1 ; a, b) & =a^{b} b \zeta(b+1, a+1)=a^{b} b \sum_{n=1}^{\infty}(n+a)^{-b-1} \\
& \geq a^{b} b \int_{1}^{\infty}(u+a)^{-b-1} d u=\left(\frac{a}{a+1}\right)^{b} .
\end{aligned}
$$

Hence $X$ has a distribution which is approximately degenerate at one when $a$ is large or $b$ is small.

Finally, it has been observed by the referee that there are two other discrete distributions $[3,10.3]$ that are related to the Riemann zeta function $\varsigma(s, 1)$.

\section{REFERENCES}

1. E. T. Whittaker and G. N. Watson, Modern analysis, Cambridge Univ. Press, New York, 1927.

2. M. Fisz, Probability theory and mathematical statistics, Wiley, New York, 1963.

3. N. L. Johnson and S. Kotz, Discrete distributions, Houghton Mifflin, Boston, Mass., 1969.

Graduate Studies and Continuing Education, Pennsylvania State UniverSity, King of PRUSSiA, PEnNSYlVANia 19406 